\title{
A Simple and Fast Method to Generate Transmit Beampattern in MIMO Radar
}

\author{
Mostafa Bolhasani $^{1}$, Sadjad Imani ${ }^{1}$, Seyed Ali Ghorashi ${ }^{1,2}$ \\ Cognitive Telecommunication Research Group, Faculty of Electrical Engineering, \\ Shahid Beheshti University G. C., Tehran, $\operatorname{Iran}^{1}$ \\ Cyber Research Centre, Shahid Beheshti University G. C., Tehran, Iran²
}

\begin{abstract}
In this paper, we consider the problem of transmit beampattern design in multiple-input multiple-output (MIMO) radar systems. MIMO systems have the potential to dramatically improve the performance of radar systems over single antenna systems and phased array radar systems. The aim of waveform design problem is to achieve a desired beampattern or concentrating the transmit power in certain directions by designing the transmit covariance matrix. In this paper we first proposed a smiple and fast method which concentrates transmit power in interested area uniformly. This method satisfy peak to average power ratio (PAPR) practical constraint of transmit power and its PAPR is equal to one. Then we proposed an other method based on first method to decrease side lobe level (SLL) of our transmit beampattern and to achieve this goal, we employ chebyshev window in our method. However, the second method decrease SLL of the transmit beampattern, but its PAPR is not equal to one and has a low-PAPR. Simulation results validate our claims.
\end{abstract}

Keywords: multiple-input multiple-output (MIMO) radar, transmit beampattern design, minimum side lobe beampattern design, chebyshev window, covariance matrix design.

\section{INTRODUCTION}

Radar is an electromagnetic system for the detection and localization of objects such as ships, aircrafts, etc.. The main principle of radar systems is to collect the electromagnetic waves from objects and process them appropriately to obtain as much information of objects as possible. The information includes the target's presence, direction, distance from a reference location, velocity, and so on. Conventional radar systems can be classified into three groups of monostatic, bistatic and multistatic [1] based on the number of antennas in the system and the distance between them. In monostatic systems, transmit and receive antennas are colocated and usually there is only one antenna operating both transmitting and receiving duties in a time multiplexed mode. In bistatic radars, there are one transmit and one receive antennas, and they are significantly separated [2]. Multistatic radars have two or more transmit or receive antennas with all antennas separated by large distances [3].

Multiple-input Multiple-output (MIMO) radars can be considered as a generalization of multistatic radar concepts. MIMO systems have the potential to dramatically improve the performance of radar systems with respect to single antenna and phased array radar systems. The concept of MIMO radar is that there are multiple transmitting \& receiving antennas and the collected information is processed together [4].

Based on the antennas configurations, MIMO radars can be grouped into two different categories; widely separated MIMO radar and coherent MIMO radar. In the first, transmit antennas of MIMO radar are widely separated [5] in order to overcome the fluctuations of target amplitude on the radar display. Both experimental and theoretical results have demonstrated that small changes in the location of target can result in a large change in the amount of energy reflected from target [6, 7]. When a large decrease in the amount of reflected energy from target is experienced, the performance of radar systems degrades, seriously. The transmit antennas in such MIMO radars are spaced widely enough such that each of them views a different aspect (scatterer) of the target. Consequently, the radar cross sections (RCS) of target are independent random variables for different transmitting paths. This widely separation of antennas can increase the spatial diversity of radar. In this type of radars, an extended target (A target that occupies more than one resolution cell) model is assumed and therefore, it takes the advantages of the associated spatially-distributed signal model. In this type of radars, the waveform diversity is similar to multi-path diversity concept in wireless communications over fading channels [8,9]. Similarly, the waveform diversity in radars enables reliable detection due to the fact that it is unlikely that all waveforms suffer from the effect of target amplitude fluctuations, simultaneously.

In the second type of radars which is called coherent MIMO radars or MIMO radars with colocated antennas [10], the antennas in both transmitter and receiver are spaced close enough so that each antenna views the same cross section of target. Thus, the RCSs of target observed by all transmit/receive propagation paths are identical, which can be considered as a deterministic unknown parameter. In these radars, target is usually assumed to be in far-field. Also, it is often assumed that the transmitted signals are narrowband and, therefore, the point source signal model is commonly considered. In this case, the waveform diversity boils down to increasing the virtual aperture of the receive array due to the fact that multiple independent waveforms are received by the same receive array [11]. Indeed, as compared to the phased-array radar, 
the use of MIMO radar with colocated antennas enable us to improve angular resolution, increase the upper limit on the number of detectable targets, to improve parameter identifiability, to extend the array aperture by virtual sensors, and to enhance the flexibility for transmit/receive beampattern design [11]-[13]. The use of colocated antennas allows the radar user to design the signal covariance matrix such that the radar antennas transmit power in certain directions in order to enhance the SINR at the receiver.

The process of waveform design can be divided into two stages; a) designing the transmit waveform covariance matrix of transmit waveforms [14-19], b) synthesizing the transmit waveforms in order to realize the designed covariance matrix of stage one [20-22]. Several algorithms are proposed in literature to design transmit waveform with different constraints such as low peak to average power ratio (PAPR), because this helps in linear behavior of amplifiers in radio frequencies [23]. A sequential algorithm is presented in [23] to jointly design the transmit waveform and receive combining filter in order to maximize the SINR for a point like target (a target whose largest physical dimension is small relative to the size of the radar resolution cell in range, angle or both) in the presence of multiple interferences based on convex optimization. This algorithm is very slow because of its high computational complexity. Thus, it is difficult to use this algorithm in practice. A sequential quadratic programming algorithm is presented in [15], which matches the desired beampattern by designing the covariance matrix. In [24] we proposed a method based on convex optimization and we jointly designed the transmit signals and the receive combining filter based on exact information on the locations of target and interferences in colocated MIMO radars. A closed form method for uniform linear array (ULA) is presented in [16], which is based on discrete Fourier transform (DFT) coefficients and Toeplitz matrix. Compared with the iterative algorithm introduced in [15], this method has a lower computational complexity, while needs a large number of antennas which usually is not practical. Hence, the methods based on iterative algorithm have high computational complexity and when the number of antennas are increasing, computational complexity of such methods based on solve optimization problem (e.g. SQP and SDP) will be high and therefore, the computational speed of these methods is slow.

In this paper, we propose a fast and simple method which can concentrate the radar transmit power around targets, uniformly. We design two covariance matrices whose transmit beampattern is made of many phased array beampatterns. Phased array radar with many transmit antennas has a beampattern with narrow width and can concentrate the transmit power in certain direction, with a high computational speed. The higher the rank of waveform covariance matrix, the higher the number of interferences that a radar can suppress. Therefore, unlike phased array covariance matrix with a rank of one, the proposed covariance matrices for MIMO radars have ranks more than one and then, can suppress more number of interferences. Both of two proposed covariance matrices have much higher rank than recent DFT-based method in [16]. This method exploits high computational speed of phased array radar and has more computational speed compared to Fourier-based method in [16] and SQP-based methods such as the one in [15]. Our second proposed method has a lower SLL compared to the Fourier-based method in [16], however, it has a low PAPR. Also, our proposed methods can transmit power to more than one interested area of space unlike the method in [25]. After designing the covariance matrix, practical signal waveforms whose covariance matrix is close to the designed covariance matrix are produced under some practical constraints $[21,22]$. Simulation results show the efficiency of our proposed methods.

The organization of this paper is as follows: In section 2 the signal model of MIMO radar is introduced and the relationship between the design of covariance matrix and transmit beampattern is shown. In section 3 our two proposed algorithms are introduced. Section 4 shows the numerical results and conclusion is provided in section 5 .

Notation: Bold upper case letters, $\mathbf{X}$, and lower case letters, $\mathrm{X}$ denote matrices and vectors, respectively. (.) $)^{T}$ and $(.)^{H}$ denote transposition and conjugate transposition, respectively, and inverse of a matrix is denoted by $(.)^{-1}$. Expectation operator is denoted by $E($.$) . The Kronecker$ product and the hadamard multiplication is denoted by $\otimes$ and $\square$, respectively. $|$.$| denotes the absolute value.$

\section{SIGNAL MODEL}

Consider a colocated MIMO radar system equipped with a transmit array of $M_{t}$ isotropic antennas. Each transmit antenna emits $s_{m}(n), m=1, \ldots, M_{t}, n=1, \ldots, L$ which is different from other transmitted waveforms, $n$ is the sample number and $L$ is the number of samples in each waveform. Also assume that

$$
\mathbf{s}(n)=\left[s_{1}(n), s_{2}(n), \ldots, s_{M_{t}}(n)\right]^{T} \text { is a } M_{t} \times 1 \text { vector of }
$$
transmit waveforms in time sample $n$. Then, the signal at target location $\theta$ would be $\mathbf{a}_{\mathrm{t}}(\theta)^{T} \mathbf{s}(n), n=1, \ldots, L$. For a ULA with half-wavelength inter-element spacing, $\mathbf{a}_{\mathbf{t}}(\theta)$ is given by

$\mathbf{a}_{\mathrm{t}}(\theta)=\left[1, \mathrm{e}^{-\mathrm{j} \pi \sin (\theta)}, \ldots, \mathrm{e}^{-\mathrm{j} \pi\left(M_{t}-1\right) \sin (\theta)}\right]^{T}$

Assume that there is a point like target at location $\theta_{0}$ as well as Q signal-dependent interference sources at locations $\theta_{j}, j=1, \ldots, Q$. Therefore, the baseband signal at receiver can be expressed as

$$
\begin{aligned}
\mathbf{y}(\mathrm{n}) & =\alpha_{0} \mathbf{a}_{\mathrm{r}}\left(\theta_{0}\right) \mathbf{a}_{\mathrm{t}}\left(\theta_{0}\right)^{\mathrm{T}} \mathbf{s}(\mathrm{n}) \\
& +\sum_{\mathrm{j}=1}^{\mathrm{Q}} \alpha_{\mathrm{j}} \mathbf{a}_{\mathrm{r}}\left(\theta_{\mathrm{j}}\right) \mathbf{a}_{\mathrm{t}}\left(\theta_{\mathrm{j}}\right)^{\mathrm{T}} \mathbf{s}(\mathrm{n})+\mathbf{v}(\mathrm{n})
\end{aligned}
$$


where $\alpha_{0}$ and $\alpha_{\mathrm{j}}$ are the RCSs of target and interference sources, respectively, and $\mathbf{a}_{\mathrm{r}}(\theta)$ represents the $M_{r} \times 1$ received steering vector. $\mathbf{v}(n)$ describes the independent and identically distributed additive white Gaussian noise vector at $M_{r}$ receivers with covariance matrix of $\sigma_{v}^{2} \mathbf{I}$. Assume also that $\alpha_{j}$ 's are mutually uncorrelated with zero mean and variance $\sigma_{j}^{2}$.

The power of the transmitted signal at target location can be expressed as

$$
\mathrm{p}(\theta)=\mathrm{E} \mid \mathbf{a}_{t}^{H}(\theta) \mathbf{s}^{2}=\mathbf{a}_{t}^{H}(\theta) \mathbf{R} \mathbf{a}_{\mathbf{t}}(\theta)
$$

where $\mathbf{R}=E\left(\mathbf{s s}^{\mathrm{H}}\right)$ is the covariance matrix of the transmitted signal. As can be seen in (3), transmit beampattern can be controlled by designing the transmit covariance $\mathbf{R}$. This matrix must be positive semidefinite because it is a covariance matrix. In waveform design problem, it is often assumed that the transmit power of all antennas are the same. The received signal at each element of receive array is passed through $M_{t}$ matched filters. The output of matched filters for $M_{r}$ receive antennas can be written as a $M_{t} M_{r} \times 1$ vector as follows

$$
\mathbf{y}=\alpha_{0} \mathbf{a}_{\mathrm{r}}\left(\theta_{0}\right) \otimes \mathbf{R}_{\mathrm{t}}\left(\theta_{0}\right)+\sum_{\mathrm{i}=1}^{\mathrm{Q}} \alpha_{\mathrm{i}} \mathbf{a}_{\mathrm{r}}\left(\theta_{\mathrm{i}}\right) \otimes \mathbf{R a}_{\mathrm{t}}\left(\theta_{\mathrm{i}}\right)+\mathbf{v}_{\mathrm{c}}
$$

where $\mathbf{v}_{\mathrm{c}}$ is the noise vector with zero mean and variance of $\sigma^{2}\left(\mathbf{I}_{M_{r}} \otimes \mathbf{R}\right)$.

Our goal is to propose a method to design a covariance matrix whose beampattern is concentrated in a certain direction. We would like our method to perform this task with a high computational speed compared to the methods based on optimization such as SQP-based method in [15] and the Fourier-based method in [16]. In the next section, we propose two methods for this; we first propose a method of designing the covariance matrix through the use of phased array covariance matrix, and then we propose another method to decrease the SLL achieved by the first proposed method.

\section{PROPOSED COVARIANCE MATRICES}

\section{A. First Proposed Covariance Matrix}

In first method, we first design a covariance matrix which is sum of phased array covariance matrices with proper step during $\theta_{1}$ to $\theta_{N}$ interval.

$$
\mathbf{R}=\sum_{i=1}^{N} \mathbf{a}_{t}\left(\theta_{i}\right) \mathbf{a}_{t}^{H}\left(\theta_{i}\right)
$$

However, when we design the covariance matrix only with sum of phased array covariance matrices, this transmit beampattern cannot cover the desired target area, uniformly. Fig. 1 shows the transmit beampattern of covariance matrix achieved by (5) for a desired area of $30^{\circ}$ width. As can be seen, the amplitude of transmit power varies dramatically for different values of $\theta$. This beampattern is not efficient, because if a target is located at a direction in which the amplitude of transmit power is low, then the reflected power from target can be not enough for detection purposes.

In order to solve this problem, we utilize these two properties a) The transmit beampattern of transpose of a covariance matrix is symmetry of that covariance matrix transmit beampattern. b) The beampattern of the covariance matrix can be shifted as follows:

$$
\mathbf{R}_{\text {shifted }}=\mathbf{R} \square\left(\mathbf{a}_{t}\left(\theta_{\text {shift }}\right) \mathbf{a}_{t}^{H}\left(\theta_{\text {shift }}\right)\right)
$$

which means that the beampattern of $\mathbf{R}_{\text {shifted }}$ is shifted with the value of $\theta_{\text {shift }}$ from the beampattern of $\mathbf{R}$.

Now, due to the properties of a and $\mathrm{b}$ mentioned above, we propose the first covariance matrix $\mathbf{R}_{1}$ as follows:

$$
\begin{gathered}
\mathbf{R}_{\text {Transposed-shifted }}=\mathbf{R}^{T} \square\left(\mathbf{a}_{t}\left(\theta_{\text {shift }}\right) \mathbf{a}_{t}^{H}\left(\theta_{\text {shift }}\right)\right)(7 \\
\mathbf{R}_{1}=\left(\mathbf{R}+\mathbf{R}_{\text {Transposed-shifted }}\right) / 2
\end{gathered}
$$

We set $\theta_{\text {shift }}$ in a way that the beampattern of the covariance matrix $\mathbf{R}_{\text {Transposed-shifted }}$ locates at the direction of the covariance matrix $\mathbf{R}$ beampattern. Then, by adding these two beampatterns together, the resulted beampattern (beampattern of $\mathbf{R}_{1}$ ) will be uniform in the desired area. The proposed algorithm is as follows:

Algorithm 1 Proposed Covariance Matrix Design

Inputs: $\theta_{1}, \theta_{N}$ and $M_{t}$

Outputs: $\mathbf{R}_{1}$ and $P(\theta)$

Set: $K=0$ and $\mathbf{R}=0$

1: Compute $\mathbf{a}_{t}(\theta)$ using (1) for $M_{t}$ antennas.

2: $\mathbf{W}\left(\theta_{K}\right)= \begin{cases}1 & \theta_{K} \in\left[\theta_{1}, \theta_{N}\right] \\ 0 & \text { O.W }\end{cases}$

3: Compute $\mathbf{R}=\mathbf{R}+\mathbf{a}_{t}(\theta) \mathbf{a}_{t}^{H}(\theta) \mathbf{W}\left(\theta_{K}\right)$

4: $K=K+1$

5: Repeat step1- step 4, until $K=180$.

6: Compute $\mathbf{a}_{t}\left(\theta_{\text {Shift }}\right)$ using (1) for $M_{t}$ antennas

7: Compute $\mathbf{R}_{\text {Transposed-shifted }}$ using (7).

8: Report $\mathbf{R}_{1}$ using (8).

9: Report $P(\theta)$ using (3).

This algorithm can design transmit beampattern with a high computational speed and satisfies the PAPR practical constraint. Also, because the rank of the proposed covariance matrix is much higher than DFT-based method in [16] and phased array radars, this method has a proper degree of freedom and can exploit the properties of MIMO radars and can suppress more interferences, potentially. 
B. Second Proposed Covariance Matrix

The second proposed algorithm is the same as the first one with a modification; we multiply a Chebyshev window to steering vector $\mathbf{a}_{t}(\theta)$ to decrease the SLL of transmit beampattern in order to better concentrate the transmit power in the desired area. In this way, the transmit powers from transmit antennas are not the same and we have a low PAPR.

Again, and similar to the first method, when we design the covariance matrix only by addition of phased array covariance matrices which are multiplied by a Chebyshev window to the mentioned steering vector, this transmit beampattern cannot cover the desired target area, uniformly. Therefore, we design a covariance matrix which is sum of the shifted-transposed of covariance matrix $\mathbf{R}^{\prime}$ and itself. The new steering vector is expressed as follows:

$$
\mathbf{b}_{t}(\theta)=\mathbf{C W} \square \mathbf{a}_{t}(\theta)
$$

where $\mathbf{C W}$ is a $M_{t} \times 1$ vector corresponding to Chebyshev window for $M_{t}$ transmit antennas. In order to employ chebyshev window, we use chebwin function in MATLAB.

The covariance matrix $\mathbf{R}^{\prime}$ is defined as follows:

$$
\mathbf{R}^{\prime}=\sum_{i=1}^{N} \mathbf{b}_{t}\left(\theta_{i}\right) \mathbf{b}_{t}^{H}\left(\theta_{i}\right)
$$

We define the two covariance matrices $\mathbf{R}_{\text {shifted }}^{\prime}$ and $\mathbf{R}_{\text {Transposed-shifted }}^{\prime}$ as follows:

$$
\begin{aligned}
& \mathbf{R}_{\text {shifted }}^{\prime}=\mathbf{R}^{\prime} \square\left(\mathbf{b}_{t}\left(\theta_{\text {shift }}\right) \mathbf{b}_{t}^{H}\left(\theta_{\text {shift }}\right)\right) \\
& \mathbf{R}_{\text {Transposed-shifted }}^{\prime}=\mathbf{R}^{\prime T} \square\left(\mathbf{b}_{t}\left(\theta_{\text {shift }}\right) \mathbf{b}_{t}^{H}\left(\theta_{\text {shift }}\right)\right)
\end{aligned}
$$

Finally, the covariance matrix is proposed as follows:

$$
\mathbf{R}_{2}=\left(\mathbf{R}^{\prime}+\mathbf{R}_{\text {Transposed-shifted }}^{\prime}\right) / 2
$$

We set $\theta_{\text {shift }}$ in a way that the beampattern of the covariance matrix $\mathbf{R}_{\text {Transposed-shifted }}^{\prime}$ locates at the direction of the beampattern of covariance matrix $\mathbf{R}^{\prime}$. Then, by adding these two beampatterns, the resulted beampattern (beampattern of $\mathbf{R}_{1}$ ) will be uniform in the desired area.

Algorithm 2 Proposed Covariance Matrix Design

Inputs: $\theta_{1}, \theta_{N}$ and $M_{t}$

Outputs: $\mathbf{R}_{2}$ and $P(\theta)$

Set: $K=0, \mathbf{R}^{\prime}=0$ and $\mathbf{C W}=\operatorname{chebwin}\left(M_{t}\right)$

1: Compute $\mathbf{b}_{t}(\theta)$ using (9) for $M_{t}$ antennas.

2: $\mathbf{W}\left(\theta_{K}\right)= \begin{cases}1 & \theta_{K} \in\left[\theta_{1}, \theta_{N}\right] \\ 0 & \text { O.W }\end{cases}$
3: Compute $\mathbf{R}^{\prime}=\mathbf{R}^{\prime}+\mathbf{b}_{t}(\theta) \mathbf{b}_{t}^{H}(\theta) \mathbf{W}\left(\theta_{K}\right)$

4: $K=K+1$

5: Repeat step1- step 4, until $K=180$

6: Compute $\mathbf{b}_{t}\left(\theta_{\text {Shift }}\right)$ using (9) for $M_{t}$ antennas.

7: Compute $\mathbf{R}_{\text {Transposed-shifted }}^{\prime}$ using (12).

8: Report $\mathbf{R}_{2}$ using (13).

9: Report $P(\theta)$ using (3).

\section{NUMERICAL RESULTS}

In this section, several numerical simulations are run in order to evaluate the efficiency of the proposed covariance matrices. In all of the following simulations, we assume that transmit arrays are uniform linear array (ULA) with half-wavelength inter-element spacing.

In the first simulation, we assume that targets are located between $\theta \in\left[10^{\circ}, 40^{\circ}\right]$ and we are going to cohere the power in these directions. Fig. 1 shows the transmit beampattern of covariance matrix in (5) for the desired area with the width of $30^{\circ}$. As can be seen, the amplitude of the transmit power varies by $\theta$ variations in the desired area and hence, this beampattern is not efficient.

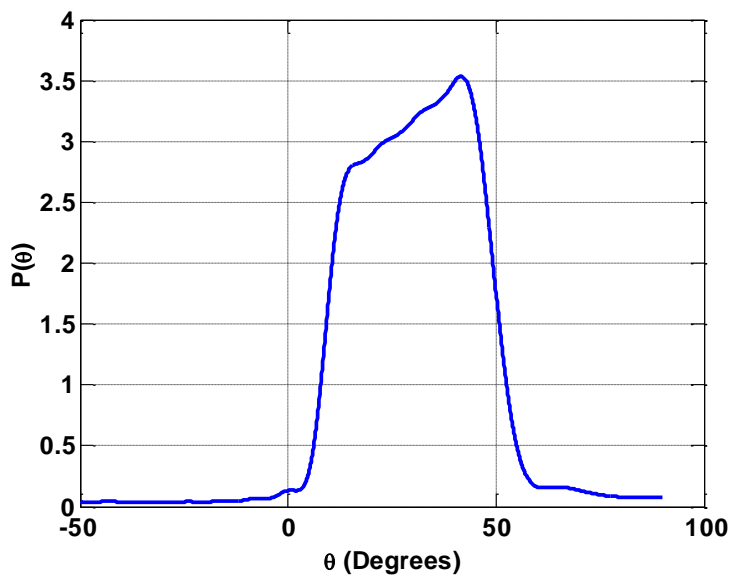

Figure 1: Transmit beampattern using the covariance matrix $\mathbf{R}$ for $M_{t}=15$ transmit antennas when beamwidth

$$
\text { is } 30^{\circ}\left(\left[10^{\circ}, 40^{\circ}\right]\right) \text {. }
$$

In the next simulation, we show the transmit beampattern of our first proposed method ( $\mathbf{R}_{1}$ ) in fig. 2. As can be seen in this figure, this transmit beampattern concentrates the transmit power in the desired area, more uniformly than what it is in the covariance matrix $\mathbf{R}$.

Now, in order to evaluate the efficiency of our proposed methods, we compared our resuts with two recently proposed methods; DFT-based method in [16] and SQPbased method in [15]. 


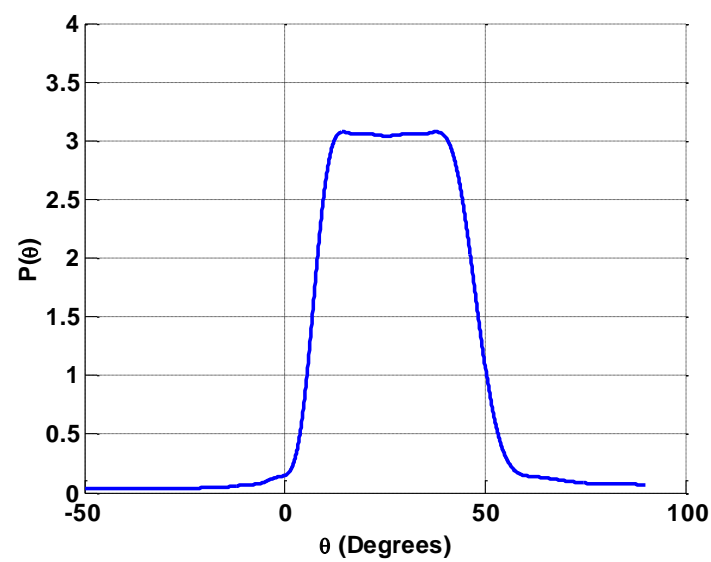

Figure 2: Transmit beampattern using the first proposed covariance matrix $\mathbf{R}_{1}$ for $M_{t}=15$ transmit antennas, when beamwidth is $30^{\circ}\left(\left[10^{\circ}, 40^{\circ}\right]\right)$.

In the next simulation, we assume a ULA with $M_{t}=15$ transmit antennas. Fig 3 shows the transmit beampattern of the first proposed method and the DFT-based method. As can be seen, unlike the DFT-based method which cannot cover the desired target area without ocillations, our proposed method can concentrate the transmit power in the desired area, uniformly. However, it has more SLL compared to the DFT-based method. It should be noted that the transmit beampattern of the DFT-based method has the same behavior as the SQP-based method.

Therefore, in the case we want to have a low SLL, we use the second proposed method which designs the covariance matrix $\mathbf{R}_{2}$. In the next simulation, we again assume a ULA with $M_{t}=15$ transmit antennas. In this simulation, the transmit beampattern of the second proposed method is compared with the DFT-based method. As can be seen in fig. 4 , when we multiply the Chebyshev window to the steering vector, our transmit beampattern losses some of its directivity but its SLL decreases, dramatically and has much lower SLL compared to the DFT-based method. However, its PAPR is not equal to one unlike the DFT-based method.

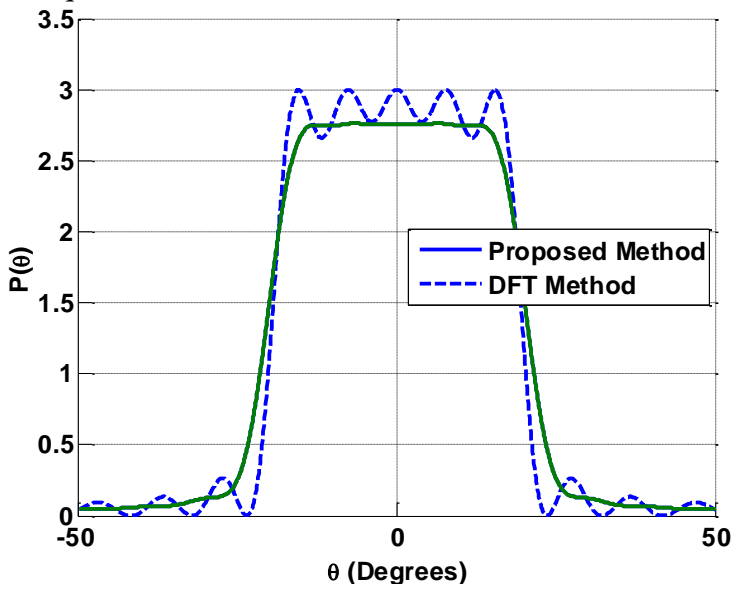

Figure 3: Comparison of transmit beampattern using the first proposed covariance matrix $\mathbf{R}_{1}$ and DFT-based method in [16] for $M_{t}=15$ transmit antennas when beamwidth is $30^{\circ}\left(\left[-15^{\circ}, 15^{\circ}\right]\right)$.
In order to demonstrate the difference in required computational time, we show a comparison of average run time required to generate the transmit beampatterns. Fig. 5 shows the average run time to generate the transmit beampattern for our proposed methods and the DFT-based method, when MATLAB is run on a personal computer with CPU:2.4 GHZ and 16GB of RAM. This figure reinforces the computational advantage of the proposed method. Also as can be seen, computational time of our proposed methods unlike DFT-based method is constant, approximately as the number of transmit antennas increases. Also, the computational time of SQP-based method is much higher than DFT-based method [16].

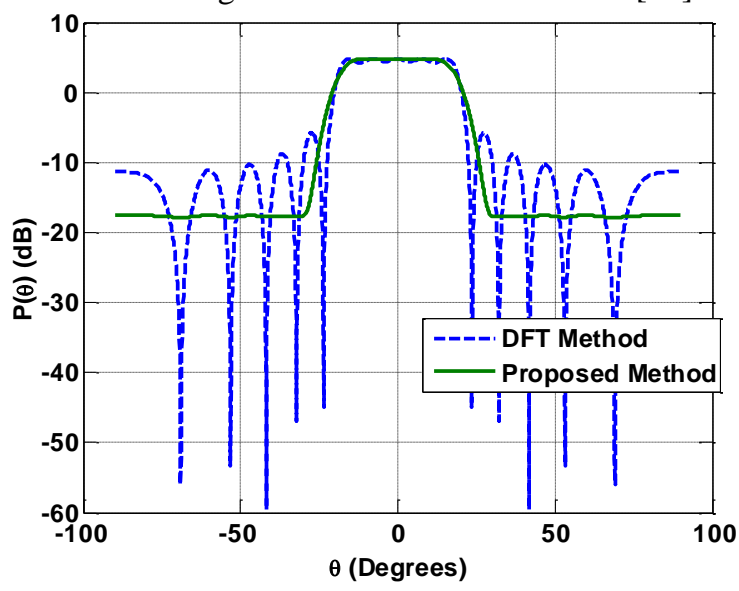

Figure 4: Comparison of transmit beampattern using the second proposed covariance matrix $\mathbf{R}_{2}$ and DFT-based

method in [16] for $M_{t}=15$ transmit antennas when beamwidth is $30^{\circ}\left(\left[-15^{\circ}, 15^{\circ}\right]\right)$.

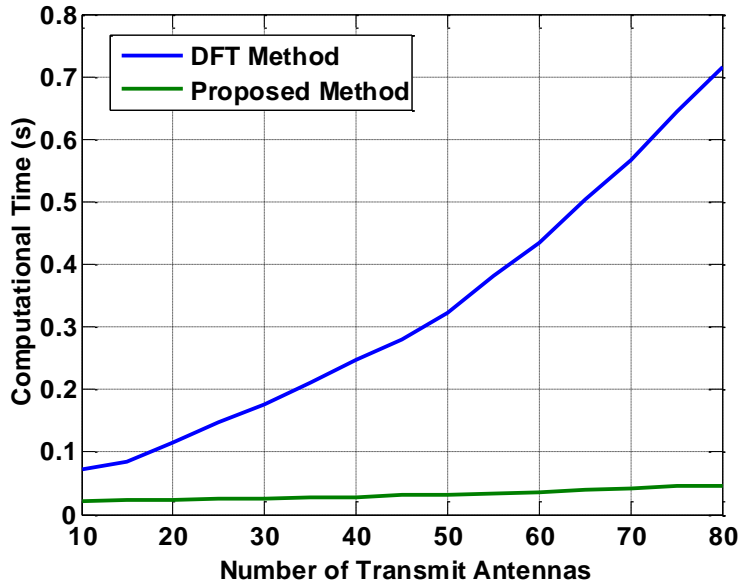

Figure 5: Computational time required to generate transmit beampattern for our proposed method and DFT-based method in [16].

In last simulation, we compared our proposed covariance matrix rank with the proposed covariance matrix rank in [16]. As can be seen in fig. 6, our proposed covariance matrix rank is more than that for proposed covariance matrix in [16]. Hence, due to the co-array concept [26], our proposed method can suppress more interferences compared to DFT-based method in [16]. As the computational time and the rank of both proposed covariance matrices are approximately the same, in our simulations we showed only the first method. 


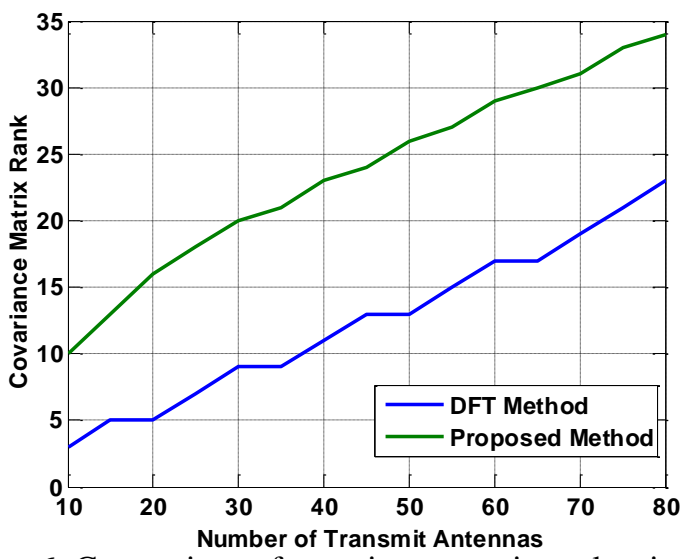

Figure 6: Comparison of covariance matrix rank using the proposed method and DFT-based method in [16].

\section{CONCLUSION}

In this paper we proposed a smiple and fast method which concentrates transmit power in interested area uniformly. This method design a covariance matrix which its rank is much higher than DFT-based method when the same number of transmit antennas is employed and can suppress more interferences potentially. Our methods have low computational time. The first proposed method satisfy peak to average power ratio (PAPR) constraint of transmit power. Then we proposed an other method based on first method to decrease side lobe level (SLL) of our transmit beampattern and to achieve this goal, we employed chebyshev window in our method. The second method decrease SLL of the transmit beampattern with low PAPR. Both of two covariance matrices can transmitting power to more than one interested area of space.

\section{REFRENCES}

[1] M. Skolnik, Introduction to Radar Systems, 3rd ed. New York: McGraw-Hill, 2002.

[2] M.A. Richards, Fundamentals of Radar Signal Processing, McGrawHill, 2005

[3] V.S. Chernyak, Fundamentals of Multisite Radar Systems : Multistatic Radars and Multiradar Systems, Gordon and Breach Science Publishers, 1998

[4] A. Merline, and S.J. Thiruvengadam, Multiple-input Multiple-output Radar Waveform Design Methodologies, Defence Science Journal, Vol. 63, No. 4, July 2013, pp. 393-401.

[5] N. Levanon, Radar Principles, 1st ed. John Wiley \& Sons, 1988

[6] D. Malioutov, M. Cetin, and A. S. Willsky, A sparse signal reconstruction perspective for source localization with sensor arrays, Signal Processing, IEEE Transactions on, vol. 53, pp. 3010-3022, 2005.

[7] H. L. V. Trees, Detection, Estimation, and Modulation Theory, vol. III. NY: John Wiley \& Sons, 1968.

[8] S. M. Alamouti, A simple transmitter diversity scheme for wireless communications, IEEE J. Sel. Areas Commun., vol. 16, pp. 1451-1458, Oct. 1998.

[9] D. Tse and P.Viswanath, Fundamentals of Wireless Communication. Cambridge, U.K.: Cambridge Univ. Press, 2005.

[10] J. Li and P. Stoica, MIMO radar with colocated antennas, IEEE Signal Process. Mag., vol. 24, no. 5, pp. 106-114, 2007.

[11] J. Li and P. Stoica, MIMO radar with colocated antennas, IEEE Signal Process. Mag., vol. 24, pp. 106-114, Sep. 2007.

12] C.-Y. Chen and P. Vaidyanathan, MIMO radar space-time adaptive processing using prolate spheroidal wave functions, IEEE Trans. Signal Process., vol. 56, no. 2, pp. 623-635, Feb. 2008.

[13] I. Bekkerman and J. Tabrikian, Target detection and localization using MIMO radars and sonars, IEEE Trans. Signal Process., vol. 54, no. 10 , pp. 3873-3883, Oct. 2006

[14] D. Fuhrmann, G. San Antonio, Transmit beamforming for MIMO radar systems using signal cross-correlation, IEEE Trans. Aerosp. Electron. Syst. 44 (1) (2008) 171-186.

[15] P. Stoica, J. Li, Y. Xie, On probing signal design for MIMO radar, IEEE Trans. Signal Process. 55 (8) (2007) 4151-4161.
[16] J. Lipor, S. Ahmed, M.-S. Alouini, Fourier-based transmit beampattern design usingMIMO radar, IEEE Trans. Signal Process. 62 (9) (2014) 2226-2235.

[17] S. Ahmed, J. Thompson, Y. Petillot, B. Mulgrew, Unconstrained synthesis of covariance matrix for MIMO radar transmit beampattern, IEEE Trans. Signal Process. 59 (8) (2011) 3837-3849.

[18] M. Soltanalian, H. Hu, P. Stoica, Single-stage transmit beamforming design for MIMO radar, Signal Processing 102 (2014) 132-138.

[19] G. Hua, S. Abeysekera, MIMO radar transmit beampattern design with ripple and transition band control, IEEE Trans. Signal Process. 61 (11) (2013) 2963-2974.

[20] Jardak, S.; Ahmed, S.; Alouini, M.-S., Generation of Correlated Finite Alphabet Waveforms Using Gaussian Random Variables, Signal Processing, IEEE Transactions on , vol.62, no.17, pp.4587,4596, Sept.1, 2014

[21] P. Stoica, J. Li, X. Zhu, Waveform synthesis for diversity-based transmit beampattern design, IEEE Trans. Signal Process. 56 (6) (2008) 2593

[22] S. Ahmed, J. Thompson, Y. Petillot, B. Mulgrew, Finite alphabet constant-envelope waveform design for MIMO radar, IEEE Trans. Signal Process. 59 (11) (2011) 5326-5337.

[23] G. Cui, H. Li, M. Rangaswamy, MIMO radar waveform design with constant modulus and similarity constraints, IEEE Trans. Signal Process. 62 (2) (2014) 343-353.

[24] Imani, S. Ghorashi, S.A., "Transmit Signal and Receive Filter Design in Co-located MIMO Radar Using a Transmit Weighting Matrix," Signal Processing Letters, IEEE, vol.22, no.10, pp.1521-1524, Oct. 2015.

[25] D. R. Fuhrmann, J. P. Browning, and M. Rangaswamy, Signaling strategies for the hybrid MIMO phased-array radar, IEEE J. Sel. Topics Signal Process., vol. 4, no. 1, pp. 66-78, Feb. 2010.

[26] J. Liu, H. Li, B. Himed, Joint optimization of transmit and receive beamforming in active arrays, Signal Processing Letters, IEEE 21 (1) (2014) 39-42.

\section{BIOGRAPHIES}

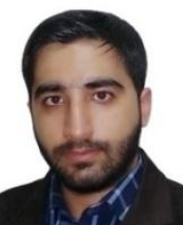

Mostafa Bolhasani received his B.Sc degree in Engineering Science/Electrical Eng. from the University of Tehran, Iran, in 2013 and is now a M.Sc student in Department of Electrical Engineering, Shahid Beheshti University G.C., at Tehran, Iran. His research is focused on MIMO radars signal processing and communication signal processing.

Sadjad Imani received his M.Sc. degree in Electrical Eng. from

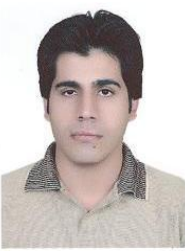
the University of Tehran, Iran, in 2011 and is now a $\mathrm{PhD}$ student in Department of Electrical Engineering, Shahid Beheshti University G.C., at Tehran, Iran. His research is focused on MIMO radars signal processing, cognitive radio and communication signal processing.

Seyed Ali Ghorashi received his B.Sc. and M.Sc. degrees in Electrical Eng. from the University of Tehran,

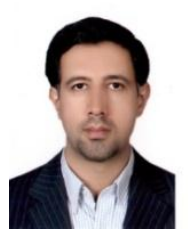
Iran, in 1992 and 1995, respectively. Then, he joined SANA Pro Inc., where he worked on modelling and simulation of OFDM based wireless LAN systems and interference cancellation methods in W-CDMA systems. Since 2000, he worked as a research associate at King's College London on "capacity enhancement methods in multi-layer W-CDMA systems" sponsored by Mobile VCE. In $2003 \mathrm{He}$ received his $\mathrm{PhD}$ at King's College and since then he worked at Kings College as a research fellow. In 2006 he joined Samsung Electronics (UK) Ltd as a senior researcher and now he is a faculty member of Cognitive Telecommunication Research Group, Department of Electrical Engineering, Shahid Beheshti University G.C., at Tehran, Iran, working on wireless communications. 